\title{
Excesso de peso e insatisfação corporal em adolescentes
}

\section{Obesity and body dissatisfaction amongst adolescents}

Maria Aparecida CONTI

Maria Fernanda Petroli FRUTUOSO²

Ana Maria Dianezi GAMBARDELLA²

\section{RE S U M O}

\section{Objetivo}

Verificar a associação entre excesso de peso e insatisfação corporal de adolescentes de uma instituição da rede particular de ensino fundamental de Santo André, SP.

\section{Métodos}

Para avaliação do excesso de peso, utilizou-se o índice de massa corporal por idade e sexo, e para a avaliação da insatisfação corporal, aplicou-se a escala de satisfação das áreas corporais.

\section{Resultados}

Participaram do estudo 147 adolescentes, sendo $35,40 \%$ do sexo masculino e $64,60 \%$ do sexo feminino. Verificou-se que $44,23 \%$ dos meninos e $18,93 \%$ das meninas apresentaram excesso de peso; $1,92 \%$ dos meninos e $3,16 \%$ das meninas, magreza, e os demais (53,85\% dos meninos e $58,98 \%$ das meninas) apresentaram estado nutricional normal. Constatou-se associação estatisticamente significante entre excesso de peso e insatisfação para as áreas do estômago, cintura e peso corporal nos meninos e para as áreas do cabelo, nádegas, quadril, coxas, pernas, estômago, ombros/costas, tônus muscular, peso e aparência geral para as meninas.

\section{Conclusão}

Meninas com excesso de peso apresentaram-se mais insatisfeitas com diversas áreas corporais, mas isso não ocorre com os meninos. É recomendável que instituições de ensino médio e fundamental desenvolvam atividades profiláticas voltadas para a conscientização da percepção pessoal e social do adolescente, assim como das pressões a que ele está compelido.

Termos de indexação: adolescente, estado nutricional, imagem corporal, obesidade.

\footnotetext{
1 Doutoranda, Departamento de Nutrição, Faculdade de Saúde Pública, Universidade de São Paulo. São Paulo, SP, Brasil.

2 Departamento de Nutrição, Faculdade de Saúde Pública, Universidade de São Paulo. Av. Dr. Arnaldo, 715, 2ํandar, 01246-904, São Paulo, SP, Brasil. Correspondência para/Correspondence to: A.M.D. GAMBARDELLA. E-mail: <gambarde@usp.br>.
} 
492 | M.A. CONTI et al.

\section{A B S T R A C T}

\section{Objective}

This study aimed at evaluating the relationship between overweight/obesity and body dissatisfaction among adolescents in a private school in Santo André, São Paulo, Brazil.

\section{Methods}

For the assessment of overweight/obesity, the body mass index for age and gender was used and for the body dissatisfaction evaluation, the body parts satisfaction scale was applied.

\section{Results}

One hundred and forty-seven adolescents were studied (35.40\% males and $64.60 \%$ females). It was shown that $44.23 \%$ of the males and $18.93 \%$ of the females were overweight/obese, $1.92 \%$ of the males and $3.16 \%$ of the females were underweight and the others were normal. A statistically significant association between body dissatisfaction and overweight/obesity was found for the stomach, waist and body weight in boys and the hair, buttocks, hips, thighs, legs, stomach, shoulder/back, muscle strength, body weight and overall appearance in girls.

\section{Conclusion}

Girls who were overweight/obese were more dissatisfied with a greater number of body areas than boys. It was recommended that middle and fundamental level schools develop prophylactic activities aimed at stimulating consciousness of personal and social perception in the adolescent and of the pressures to which he is submitted.

Indexing terms: adolescent, nutritional status, body image, obesity.

\section{N T R O D U Ç Ã O}

A Organização Mundial de Saúde (OMS) ${ }^{1}$ define adolescência como o período compreendido entre dez e dezenove anos de idade. Nessa etapa, o crescimento e o desenvolvimento físico são fortemente influenciados pela interação de fatores genéticos e ambientais² .

Ao chegar à adolescência, o indivíduo traz consigo os efeitos de uma interação herança-ambiente anterior que, se tiver sido desfavorável, não permitirá o pleno desenvolvimento de seus potenciais. Tomado por um turbilhão de transformações, o adolescente comumente passa a ter dificuldades de entender e lidar com seu novo universo físico e mental. Em meio às transformações hormonais, funcionais, afetivas e sociais, as alterações de seu universo físico (corporal) adquirem importância fundamental ${ }^{3}$.

Atualmente, observa-se uma série de distúrbios nutricionais em adolescentes, caracterizados tanto pelo excesso quanto pelo déficit nutricional ${ }^{1,4,5}$
Dados recentes comprovam o aumento da prevalência de obesidade entre os adolescentes. Wang et al. ${ }^{6}$ estudaram a tendência de obesidade e baixo peso em crianças e adolescentes, constatando aumento significativo de prevalência de obesidade. No Brasil ${ }^{6}$, a prevalência de obesidade entre os jovens aumentou em 8,9\% no período de 1974-1997.

Entre as conseqüências da obesidade em adolescentes, encontram-se maiores probabilidades de ser um adulto obeso e de desenvolver doenças como hipertensão arterial, dislipidemias, diabetes tipo 2, além de problemas respiratórios, musculares, baixa auto-estima, dificuldade de relacionamento entre os pares e piora da qualidade de vida ${ }^{1,4,7}$.

Independentemente do gênero, os adolescentes preocupam-se com peso corporal e aparência. Brook \& Tepper ${ }^{8}$ apontam que essa preocupação pode estar relacionada aos transtornos alimentares e obesidade. Hábitos alimentares inadequados, associados a doenças, constituem potenciais problemas de saúde pública. Fowler ${ }^{9}$, pesquisando adolescentes 
norte-americanos sobre a percepção do peso corporal, constatou, para o grupo classificado com sobrepeso/obesidade, que apesar da consciência sobre o peso, apresentavam baixa auto-estima e alto índice de insatisfação corporal.

Estudo longitudinal desenvolvido por Attie \& Brooks-Gunn ${ }^{10}$ indicou a insatisfação corporal como o único fator significante, entre outros estudados, que prognosticou o desenvolvimento de futuros problemas de ordem alimentar ${ }^{11}$.

A distorção da percepção corporal, ou seja, superestimar ou subestimar o tamanho e/ou forma do corpo, não constitui característica particular de adolescentes que desenvolvem algum tipo de transtorno alimentar, uma vez que se torna cada vez mais presente na dinâmica vivencial dos indivíduos dessa faixa etária. Fatores sociais, influências socioculturais, pressões da mídia e a busca incessante por um padrão de corpo ideal associado às realizações e felicidade estão entre as causas das alterações da percepção da imagem corporal, gerando insatisfação em especial para indivíduos do gênero feminino.

Considerando as conseqüências adversas decorrentes dos transtornos alimentares, bem como da obesidade, os profissionais da saúde devem se comprometer não só com os aspectos curativos voltados ao tratamento, mas também, e principalmente, com os aspectos preventivos, evitando, assim, que futuramente outros jovens possam desenvolver patologias semelhantes, tendo como conseqüência o bloqueio do seu desenvolvimento global.
Ainda que este tema tenha sido estudado em diversos países (Brook \& Tepper ${ }^{8}$; Kostanski \& Gullone ${ }^{11}$; Cuadrado et al. ${ }^{12}$; Rosenblum \& Lewis ${ }^{13}$ ), ignora-se registro científico nacional, fato que nos motivou a investigar a associação entre excesso de peso e insatisfação corporal de um grupo de adolescentes, de ambos os sexos.

\section{MÉ T O D O S}

O estudo foi realizado em uma instituição da rede particular de ensino fundamental localizada no município de Santo André, SP.

A amostra era constituída de 187 indivíduos que representaram $50 \%$ do total de alunos da instituição, participando do estudo 147 adolescentes. Os dados foram obtidos por meio de um questionário respondido pelo próprio aluno.

A população de estudo constituiu-se por adolescentes, de ambos os sexos, de dez a catorze anos de idade. Para a seleção da amostra adotou-se o critério de amostra casual simples ${ }^{14}$.

Para avaliação do excesso de peso dos adolescentes utilizou-se o índice de massa corporal (IMC) (peso/estatura²) por idade e sexo, adotando-se como critério os valores referentes aos percentis 5 (para magreza) e 85 (para sobrepeso e obesidade) para população norte-americana' (Quadro 1).

Os dados antropométricos (peso e estatura) foram coletados segundo método proposto por Gordon et al. ${ }^{15}$, utilizando-se, para a medição de peso corporal, a balança eletrônica do tipo plata-

Quadro 1. Percentis do índice de massa corporal para adolescentes segundo idade (em anos) e sexo. Santo André, SP.

\begin{tabular}{|c|c|c|c|c|c|c|c|c|c|c|}
\hline \multirow{3}{*}{ Idade (anos) } & \multicolumn{10}{|c|}{ Percentis } \\
\hline & \multicolumn{2}{|c|}{5 th } & \multicolumn{2}{|c|}{ 15th } & \multicolumn{2}{|c|}{ 50th } & \multicolumn{2}{|c|}{ 85th } & \multicolumn{2}{|c|}{ 95th } \\
\hline & M & $\mathrm{F}$ & $M$ & $\mathrm{~F}$ & $M$ & $\mathrm{~F}$ & M & $\mathrm{F}$ & M & $\mathrm{F}$ \\
\hline 10 & 14,42 & 14,23 & 15,15 & 15,09 & 16,72 & 17,00 & 19,60 & 20,19 & 22,60 & 23,20 \\
\hline 11 & 14,83 & 14,60 & 15,59 & 15,53 & 17,28 & 17,67 & 20,35 & 21,18 & 23,73 & 24,59 \\
\hline 12 & 15,24 & 14,98 & 16,06 & 15,98 & 17,87 & 18,35 & 21,12 & 22,17 & 24,89 & 25,95 \\
\hline 13 & 15,73 & 15,36 & 16,62 & 16,43 & 18,53 & 18,95 & 21,93 & 23,08 & 25,93 & 27,07 \\
\hline 14 & 16,18 & 15,67 & 17,20 & 16,79 & 19,22 & 19,32 & 22,77 & 23,88 & 26,93 & 27,97 \\
\hline
\end{tabular}

Fonte: World Health Organization'; $M=$ masculino; F= feminino. 
forma com capacidade para 150kg e graduação em 100g (TANITA TBF-521) e para a estatura, estadiômetro (SECA), com escala em milímetros, fixado à parede. Realizaram-se duas medidas de estatura e considerou-se a média dos valores obtidos.

Para avaliar a insatisfação por áreas corporais aplicou-se a escala proposta e validada por Brown et al. ${ }^{16}$ e adaptada por Loland ${ }^{17}$, que consta de quinze itens, a saber: rosto, cabelo, nádegas, quadril, coxas, pernas, estômago, cintura, seio/ tórax, costas/ombros, braços, tônus muscular, peso, altura, todas as áreas. Solicitou-se a cada adolescente assinalar seu grau de satisfação em relação a cada área mencionada, segundo a escala de variação: muito insatisfeito (1) a muito satisfeito (5). Para verificar as relações de associação entre as variáveis, utilizou-se o teste qui-quadrado $\left(\chi^{2}\right)$, adotando-se nível de significância de $5 \%$.

O projeto foi aprovado pelo Comitê de Ética em Pesquisa de uma Instituição de Ensino Superior e os pais e/ou responsáveis firmaram termo de consentimento para participação da pesquisa.

\section{RESULTADOS}

Observou-se que o tema da pesquisa não despertou nem interesse nem curiosidade por parte dos meninos, mesmo após explicação detalhada realizada em sala de aula, uma vez que apenas $58 \%$ deles participaram da pesquisa. Para o grupo feminino, foi motivo de perguntas e controvérsias, revelando a atenção das adolescentes para o tema pesquisado, tendo participado do estudo $98 \%$ das meninas selecionadas. Outro aspecto que pode ter interferido no desinteresse dos meninos diz respeito ao fato de os dados terem sido coletados durante a aula de educação física, a predileta para a maioria dos meninos.

Participaram do estudo 147 adolescentes, sendo $35,4 \%$ do sexo masculino e $64,6 \%$ do sexo feminino, na faixa etária de dez a catorze anos, inclusive. A média de idade para ambos os sexos foi de doze anos com desvio-padrão de 1,2 e 0,9 , respectivamente, para meninos e meninas. A maioria (95,8\%) encontrava-se no grupo etário entre onze e treze anos de idade.

Detectou-se em 18,9\% das meninas sobrepeso e obesidade, classificação que entre os meninos mostrou-se praticamente duplicada $(44,2 \%)$. Registrou-se magreza entre 1,9\% dos meninos e 3,1\% das meninas, e os demais apresentaram estado nutricional normal. No entanto, cabe lembrar que o valor elevado de índice de massa corporal (IMC) não representa necessariamente obesidade, caracterizada pelo excesso de gordura corporal. Podem refletir as alterações na composição corporal relacionada tanto ao aumento de massa magra quanto da massa gorda, características da puberdade.

Os demais adolescentes apresentaram valor de IMC considerado normal e em pequena proporção registrou-se magreza (1,92\% dos meninos e $3,16 \%$ das meninas).

Detectou-se entre os meninos associação estatisticamente significante entre excesso de peso e insatisfação corporal, para as áreas as quais se observa acúmulo de gordura (estômago e cintura), bem como em relação ao peso corporal; nas meninas, para áreas do cabelo, nádegas, quadril, coxas, pernas, estômago, ombros/costas, tônus muscular, peso corporal e aspectos gerais (Tabela 1).

\section{I S C U S S Ã O}

Tanto meninos quanto meninas reportaram insatisfação para a área do estômago e para o peso corporal. No entanto, para as meninas o excesso de peso afeta até o cabelo (Tabela 1).

Alguns estudos foram desenvolvidos na tentativa de entender a complexa dinâmica vivencial dos adolescentes. O'Dea \& Caputi ${ }^{18}$, pesquisando imagem corporal, constataram que meninas com sobrepeso/obesidade percebiam-se maiores, comparando-se aos meninos com mesmo estado nutricional. 
Tabela 1.Distribuição em porcentagem dos adolescentes com sobrepeso e obesidade, segundo insatisfação corporal e áreas corporais. Santo André, SP.

\begin{tabular}{|c|c|c|c|c|c|c|}
\hline \multirow{3}{*}{ Áreas corporais } & \multicolumn{6}{|c|}{ Sobrepeso/Obesidade } \\
\hline & \multirow{2}{*}{$\frac{\text { Meninos }(\mathrm{n}=23 \text { ) }}{\text { Insatisfeitos }}$} & \multicolumn{2}{|c|}{ Teste } & \multirow{2}{*}{$\frac{\text { Meninas }(\mathrm{n}=18)}{\text { Insatisfeitos }}$} & \multicolumn{2}{|c|}{ Teste } \\
\hline & & $\chi^{2}$ & $p$ & & $\chi^{2}$ & $p$ \\
\hline Rosto & 4,2 & 4,19 & $<0,12$ & 9,5 & 0,37 & $<0,83$ \\
\hline Cabelo & 4,2 & 0,16 & $<0,92$ & 23,8 & 9,48 & $<0,008$ \\
\hline Nádegas & 20,8 & 4,15 & $<0,12$ & 23,8 & 9,3 & $<0,01$ \\
\hline Quadril & 16,7 & 2,77 & $<0,25$ & 66,7 & 20,24 & $<0,001$ \\
\hline Coxas & 37,5 & 5,34 & $<0,06$ & 33,3 & 12,69 & $<0,001$ \\
\hline Pernas & 12,5 & 1,48 & $<0,47$ & 33,3 & 10,52 & $<0,005$ \\
\hline Estômago & 45,8 & 12,26 & $<0,002$ & 38,1 & 9,68 & $<0,007$ \\
\hline Cintura & 37,5 & 11,09 & $<0,003$ & 23,8 & 5,35 & $<0,006$ \\
\hline Tórax/Seio & 12,5 & 4,43 & $<0,10$ & 23,8 & 1,29 & $<0,52$ \\
\hline Ombro/Costas & 4,2 & 1,67 & $<0.43$ & 14,3 & 5,83 & $<0,05$ \\
\hline Braços & 16,7 & 0,03 & $<0,98$ & 9,5 & 3,33 & $<0,18$ \\
\hline Tônus muscular & 20,8 & 2,61 & $<0,27$ & 23,8 & 9,57 & $<0,008$ \\
\hline Peso & 62,5 & 21,72 & $<0,001$ & 71,4 & 21,28 & $<0,001$ \\
\hline Altura & 8,3 & 1,21 & $<0,54$ & 19,0 & 0,94 & $<0,62$ \\
\hline Aspectos gerais & 4,2 & 2,73 & $<0,25$ & 9,5 & 7,31 & $<0,02$ \\
\hline
\end{tabular}

$p<0,005$.

Richards et al. ${ }^{19}$ verificaram nos adolescentes com baixo peso, média satisfação corporal; nos de peso normal, alta satisfação, e naqueles com sobrepeso, baixa satisfação corporal, indicando associação entre estado nutricional e satisfação corporal. Achados semelhantes foram reportados por Rosemblum \& Lewis ${ }^{15}$, que detectaram associação entre insatisfação corporal e sobrepeso. Byrne \& Hills ${ }^{20}$ verificaram em adolescentes obesos de onze a dezessete anos de idade, maior insatisfação corporal quando comparados aos seus pares classificados como normais.

Rand \& Resnick ${ }^{21}$, pesquisando a aceitação do tamanho corporal de adolescentes, verificaram maior insatisfação entre meninas do que meninos, sendo $46 \%$ dos obesos insatisfeitos com o tamanho corporal.

O excesso de peso parece oferecer aos jovens satisfações específicas com possíveis atitudes e reações peculiares. Na cultura ocidental, ser magra, para mulher, simboliza competência, sucesso, controle e atrativos sexuais, enquanto excesso de peso e obesidade representa preguiça, indulgência pessoal e falta de autocontrole e força de vontade ${ }^{22}$. Sendo assim, o excesso de peso oferece uma conotação pejorativa às adolescentes, sendo, possivelmente, uns dos fatores explicativos para a insatisfação feminina.

Essa insatisfação com o tamanho corporal gera, especialmente nas meninas, o desejo de ser mais magra. Brook \& Tepper ${ }^{10}$, estudando esse aspecto perceptivo entre adolescentes de catorze a dezoito anos de idade, constataram que esse desejo se mostra quatro vezes superior nas meninas quando comparado com o desejo entre os meninos.

Nas sociedades ocidentais observam-se, entre adolescentes, atitudes algumas vezes sem fundamento a fim de emagrecer, de diminuir o tamanho do corpo. Brook \& Tepper $^{10}$ verificaram que de um total de 141 adolescentes norte-americanos, $44 \%$ percebiam-se obesos, ainda que, de fato, apenas $10 \%$ se encontrassem com sobrepeso.

Estudando as diferenças psicossociais associadas ao peso e satisfação corporal, Pesa et al. ${ }^{23}$ constataram $21 \%$ das adolescentes com sobrepeso e obesidade revelando sofrer de baixa auto-estima, fato não evidenciado nas demais. 
A associação entre excesso de peso e insatisfação por áreas corporais foi constatada na literatura. Os registros científicos apontam que a insatisfação corporal para o gênero feminino expressa-se de forma mais acentuada quando comparada ao masculino, sendo esse fato também observado no presente estudo. Algumas peculiaridades presentes na vivência do adolescente elucidam tal diferença. Os aspectos sociais foram estudados por Nowak ${ }^{24}$, que concluiu que insatisfações para distintas áreas corporais têm diferentes origens, refletindo atitudes sociais. Meninos desejam corpo adulto masculino com ombro largo, cintura fina, quadril fino e estômago plano; meninas, em contraponto, almejam corpos pequenos, com coxas, nádegas, cintura e estômago delgados.

A cultura e os avanços tecnológicos ocidentais apresentam-se particularmente responsáveis em promover esse padrão de beleza magro e de reforçar crenças culturais sobre a importância do controle de peso 22 .

Para Rosenblum \& Lewis ${ }^{15}$, independentemente do estado nutricional, as meninas desejam ser mais magras, em contrapartida jovens com sobrepeso e obesidade almejam emagrecer e aqueles com baixo peso desejam aumentar o tamanho corporal ${ }^{13}$. Ricciardelli et al. ${ }^{25}$ constataram que as influências socioculturais afetam diferentemente meninos e meninas. Os primeiros são estimulados a praticarem atividades esportivas enquanto meninas a praticarem atividades que impliquem perda de peso. Isso significa que os meninos estão sendo protegidos, uma vez que são estimulados a praticar atividades que desenvolvem outras competências, como as afetivo-cognitivas e sociais, o mesmo não ocorrendo com as meninas, que são estimuladas a praticar atividades individuais, com enfoque no caráter estético.

Outro fator a ser observado se refere à pressão da mídia. Martin \& Gentry ${ }^{26}$ constataram o efeito negativo das campanhas publicitárias em revistas de moda em adolescentes de dez a catorze anos de idade, provocado pela compa- ração dos próprios atrativos físicos com os dos modelos dos anúncios. Dessa forma, as meninas apresentaram-se mais vulneráveis ao desenvolvimento e reforço da insatisfação corporal.

\section{O N CLUS Ã O}

Meninas com excesso de peso demonstraram maior insatisfação, quando comparadas aos seus pares e aos meninos.

Investigações em saúde pública apontam que os possíveis agentes motivadores da insatisfação corporal dos adolescentes sejam a pressão da mídia e a influência social. São necessários maiores estudos nessa linha, a fim de investigar e entender a complexa dinâmica individual e social do adolescente.

Recomenda-se que as escolas destinem espaço a um profissional de saúde para o desenvolvimento de atividades profiláticas voltadas para a conscientização da percepção pessoal e social do adolescente, bem como das pressões a que está compelido.

\section{RE FER Ê N CIAS}

1. World Health Organization. Physical status: use and interpretation of anthropometry. Genova: WHO; 1995.

2. Colli A. Conceito de adolescência. In: Marcondes E. Pediatria básica. 8.ed. São Paulo: Sarvier; 1992.

3. Fleitlich BW. O papel da imagem corporal e os riscos de transtornos alimentares. Pediatr Mod. 1997; 32(1-2):56-62.

4. Dietz WH. Health consequences of obesity in youth: childhood predictors of adult disease. Pediatrics. 1998; 101(3 Pt2):518-25.

5. Nunes MAA, Pinheiro AP. Risco e prevenção em transtornos do comportamento alimentar. In: Transtornos Alimentares e Obesidade. Porto Alegre: Artes Médicas; 1998.

6. Wang Y, Monteiro C, Popkin BM. Trends of obesity and underweight in older children and adolescent in the United States, Brazil, China and Russia. Am J Clin Nutr. 2002; 75(6):971-7.

7. Himes J, Dietz WH. Guidelines for overweight in adolescent preventive service: recommendations 
from an expert committee. Am J Clin Nutr. 1994; 59(2):307-16.

8. Brook U, Tepper I. High school students' attitudes and knowledge of food consumption and body image: implications for school based education. Patient Educ Couns. 1997; 30(3):283-8.

9. Fowler BA. The relationship of body image perception and weight status to recent change in weight status of adolescent female. Adolescence. 1989; 24(95):557-68.

10. Attie I, Brooks-Gunn J. Development of eating problems in adolescent girls: a longitudinal study. Dev Psychol. 1989; 25:70-9.

11. Kostanski M, Gullone E. Adolescent body image dissatisfaction: Relation with self-esteem, anxiety, and depression controlling body mass. I Child Psychol Psychiatric. 1998; 39(2):255-62.

12. Cuadrado C, Carbajal A, Moreiras O. Body perceptions and slimming attitudes reported by Spanish adolescents. Eur J Clin Nutr. 2000; 54(1):65-8.

13. Rosenblum GD, Lewis $M$. The relations among body image, physical attractiveness, and body mass in adolescence. Child Dev. 1999; 70(1):50-64.

14. Guedes MLS, Guedes IS. Bioestatística para profissionais de saúde. Rio de Janeiro: Livro Técnico; 1988.

15. Brown TA, Cash TF, Mikulka PJ. Attitudinal body-image assessment: factor analysis of the body-self relations questionnaire. J Pers Assess. 1990; 55(1-2):135-44.

16. Loland NW. Body image and physical activity. A survey among Norwegian men and women. Int J Sport Psychol. 1998; 29:339-65.

17. Gordon CC, Chumlea WC, Roche AF. Stature, recumbent length, and weight. In: Lohman TG, Roche AF, Martorell R. Anthropometric standardization reference manual. Champaing: Human Kinetics Books; 1988.
18. O'Dea JA, Caputi P. Association between socioeconomic status, weight, age and gender, and the body image and weight control practices of 6- to 19- year-old children and adolescent. Health Educ Res. 2001; 16(5):521-32.

19. Richards MH, Petersen AC, Boxer AM, Albrecht R. Relation of weight to body image in pubertal girls and boys from two communities. Dev Psychol. 1990; 26(2):313-21.

20. Byrne NM, Hills AP. Correlations of body composition and body-image assessments of adolescents. Percept Mot Skills. 1997; 84(3 Pt 2):1330-6.

21. Rand CS, Resnick JL. The "good enough" body size as judged by people of varying age and weight. Obes Res. 2000; 8(4):309-16.

22. Wilfley DE, Rodin J. Cultural influences on eating disorders. In: Brownell KD, Fairburn CG. Eating disorders and obesity: a comprehensive handbook. New York: Guilford Press; 1995. p.135-49.

23. Pesa JA, Syre TR, Jones E. Psychosocial differences associated with body weight among female adolescents: the importance of body image. J Adolesc Health. 2000; 26(5):330-7.

24. Nowak M. The weight-conscious adolescent. J Adolesc Health. 1998; 23(6):389-98.

25. Ricciardelli LA, McCabe MP, Banfield S. Sociocultural influences on body image and body changes methods. J Adolesc Health. 2000; 26(1): 3-4.

26. Martin MC, Gentry JW. Stuck in the model trap: the effects of beautiful models in ad on female pre-adolescents and adolescents. J Advertising. 1997; 26(2):19-33.

Recebido para publicação em 6 de fevereiro de 2003 e aceito em 15 de dezembro de 2004. 